\title{
61
}

\section{A Review of the First Cooperative Projects in the Italian $\boldsymbol{e}$-Government Initiative}

\author{
Massimo Mecella ${ }^{1}$ and Carlo Batini ${ }^{2}$ \\ 'Dipartimento di Informatica e Sistemistica, Università di Roma "La Sapienza", Via Salaria \\ 113, I-00198 Roma, Italy, mecella@dis.uniromal.it \\ ${ }^{2}$ Autorità per l'Informatica nella Pubblica Amministrazione, Via Isonzo 21B, I-001 98 Roma, \\ Italy, batini@aipa.it
}

\begin{abstract}
The Italian approach to $e$-Government is based on the development and deployment of the Unitary Network, a "secure Intranet" interconnecting all the public administrations. The Cooperative Architecture, currently designed on top of it, is the reference distributed computing model in which each administration is represented as a Domain, exchanging data and application services with the others through Cooperative Gateways. Some issues have been, and currently need to be, addressed, such as the presence of legacy systems, the need of a cooperative development process, the identification of the more effective cooperative approaches. In this paper some first experimental projects carried out in the years 1998-2000, aiming at validating the Cooperative Architecture, will be described; then some lessons gained by these first experiences will be presented.
\end{abstract}

\section{INTRODUCTION}

In Italy, the need for a better coordination of efforts and investments in the area of government information systems has pushed, in 1993, the Italian Parliament to create a new agency named "Autorità per l'Informatica nella Pubblica Amministrazione" [Authority for Information Technology in the Public Administration] (AIPA) with the aim of promoting technology innovation, by defining criteria for planning, implementation, management and maintenance of the information systems of the Italian Public Administration (PA). Among the various initiatives undertaken by AIPA since its constitution, the Unitary Network is the most important and challenging one. The project has the purpose of implementing a "secure Intranet" able to connect public administrations among them. 
The ambitious objectives of the Unitary Network will be obtained by promoting cooperation at the application level. By defining a common application architecture, the Cooperative Architecture, it will be possible to consider the set of distributed, yet independent systems of public administrations as a Unitary Information System of Italian PA in which each subject can participate by exchanging services with other subjects.

The Unitary Network and the related Unitary Information System with its Cooperative Architecture are an example of a Cooperative Information System (CIS) (Mylopoulos and Papazoglou 1997, Brodie 1998); a CIS is defined as a large number of cooperating component systems distributed over large, complex computer and communication networks and working together cooperatively, effectively requesting and sharing information, constraints, and goals. The technologies supporting this kind of systems are quickly growing, but the same is not true for the methodologies on how to develop them; moreover not many experiences on successful large cooperative systems have been reported.

The aim of this paper is to describe the Cooperative Architecture and some pilot projects undertaken during the last years (1998-2000), by introducing several criteria for comparing them. The remainder of this paper is organized as follows. In Section 2 a general assessment on the use of IT in the Italian PA during the 90's is described, then the Unitary Network and the Cooperative Architecture are outlined. Section 3 describes the pilot projects and compares them by introducing some criteria. Finally Section 4 concludes the paper.

\section{THE UNITARY NETWORK}

\subsection{The assessment}

The need of a common infrastructure for connecting information systems of different public administrations has been confirmed by the results of a general assessment on the use of the Information Technology in the Italian PA. In order to perform such an assessment, AIPA has developed its own original methodology, tailoring existing approaches and methodologies for information system planning (U.S. Department of Commerce 1988) and inventory organization (Thompson 1993) to the specific features and rules of the Italian PA.

The novelty of the investigation has been to focus not only on technological issues, but also on organizational ones, to identify the structure and the relationships among the organizational units (OUs); the investigation has been performed both on the OUs responsible for the computer-based information systems (EDP OUs), and on the other units responsible for administrative processes (User OUs). The results of the investigation have been collected in an inventory (Batini et al. 1996), whose high level schema is shown in Figure. 


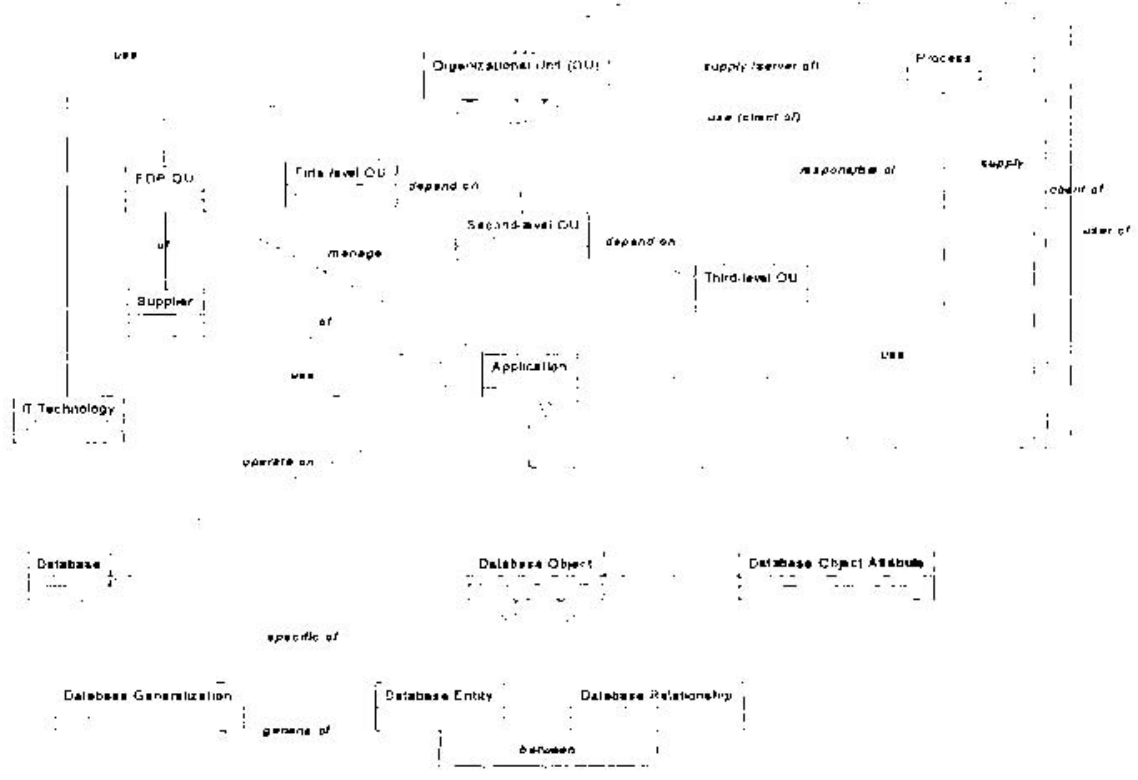

Figure 1. Schema of the investigation (in UML)

As regards the organizational structure, the Italian PA consists of different entities:

- Central PA, which consists of government Departments and Agencies (about 100, with 40.000 employees) located in Rome;

- Peripheral PA, which consists of branch offices (about 4.000 with 400.000 employees) of the Central PA spread all over the country;

- Local PA, which consists of entities such as Regions, Districts and City Councils (20.000 with 1.000 .000 employees), characterized by a high degree of autonomy with respect to the central PA; this autonomy is continuously increasing as a consequence of the decentralization process presently going on.

All together the administrations provide services to customers (citizens and private organizations such as companies, enterprises, no-profit organizations, etc.) and therefore they are typically involved together in the execution of administrative activities or processes. By analyzing processes, AIPA has tried to build macroprocesses, that is sequences or aggregations of processes that are to be executed jointly in order to satisfy the request of a service from a customer (Mecella and Batini 2001). The investigation has been performed with a bottom up strategy, leading to the identification of single administrative activities in specific OUs. Such activities, when related in terms of causal relationships, give rise to macroprocesses. 
Currently the lack of cooperation among subjects involved in macro-processes lead to their inefficient execution and to poor provision of services to customers; the development of an Unitary Information System of the PA will allow to automatize such macro-processes (Mecella and Batini 2001).

\subsection{The Architecture}

The Unitary Network architecture consists of three functional elements, for Transport, Basic and Cooperative Services (Mecella and Batini 2001). The Cooperative Service includes the middleware services that enable the development and deployment of new cooperative applications among administrations; the Cooperative Architecture is the distributed computing model based upon the Cooperative Service.

The main concept underlying the Cooperative Architecture is the one of Domain; a Domain includes all the computing resources, networks, applications and data that belong to a specific administration, regardless of the technical nature of such information system. Each such Domain is modeled as a single entity, regardless of its internal complexity, and is connected to the Unitary Network through the Domain Cooperative Gateway, that exports, through Cooperative Interfaces, the set of data and application services offered by the Domain. The interfaces may be designed according to different paradigms and modeling approaches, and deployed through various technologies. In the last three years (1998-2000) different pilot projects have been experimenting different solutions:

- by using a middleware-based approach; that is a collection of business objects and components is first specified using an appropriate Interface Definition Language, then implemented in Cooperative Gateways, and finally made available to other Domains by deploying the Gateways as object/component servers, such as CORBA, COM+ or EJB servers (mainly through protocols over TCP/IP, e.g. IIOP) (Mecella and Batini 2000);

- by using a Web-based approach; that is the data are described and exported through XML documents and the exchange is carried out through standard Internet technologies and protocols (mainly HTTP);

- by using a traditional approach; that is the data and application services are described as procedural calls and the adopted technologies are the consolidated ones (e.g. file transfer, widespread proprietary protocols, TP monitors, etc.).

In the next section such cooperative projects will be presented and compared according to some proposed dimensions. 
Six different projects will be considered:

- Arconet aims at developing a prototype of the Cooperative Gateway of the Italian Social Security Service (ISSS), for exporting data and services about pension plans and pre-retirement contributions of the Italian citizens. Two prototypes have been developed, the first by using a middleware-based approach (implemented both in DCOM and in CORBA), the latter by using XML and software components (EJBs and COM+ components). Both the prototypes use a wrapping layered architecture, in order to offer new integrated views of the data and services available on the back-end legacy systems (see Mecella and Batini 2000 for technical details).

- SICAP aims at implementing the Cooperative Information System of the Department of Justice (DoJ). The architecture and modeling approach are the same as in the first ARCONET prototype (middleware-based approach by using CORBA objects for exporting data and services). The interesting aspect is the use of a nested architecture, that is the Domain has been split into sub-domains (Criminal Record Office, many Law Courts, etc.) and each sub-domain offers a cooperative gateway similar to the main one exposed on the Unitary Network.

- WebArch aims at implementing a subsystem of the Prefectures (PreFs) concerning the Document Register (the act of recording every inward/outward documents, as defined by the Italian law) and the workflow. This subsystem was developed from scratch and it was completely written in Java, according to a component architecture quite similar to the EJB one. The interfaces towards the Unitary Network are deployed as a set of CORBA wrappers over the back-end Java components.

- $\boldsymbol{R} \boldsymbol{A} \boldsymbol{E}$ is a system for the notification of events concerning Italian enterprises, to be exchanged among the Chambers of Commerce (CsoC), ISSS and the National Board for the Insurance against Industrial Accidents (NBIIA). This system is going to implement a Publish\&Subscribe pattern, deployed by using Message Oriented Middleware (MOM) and XML DTD/documents for the definition and exchange of events. This project is the only one providing for an explicit activity, in the development process, which addresses the data quality issue.

- SICC is a system for cadastral data exchange among City Councils (CCs), Department of Finance (DoF), Notaries and Certified Land Surveyors. It uses the Access Keys Warehouse (AKW) approach (Arcieri et al. 1999), that is a warehouse of database identifiers is used to maintain the coherence among the distributed databases of the cooperative system.

- Digital Order of Payment is an e-procurement project for the development of a system managing orders of payment among public administrations (e.g., Department of Treasury, DoT) banks and suppliers. The system will be developed in two steps: (i) the deployment, in each administration, of a special- 
purpose cooperative gateway, and (ii) the development and deployment, in each administration, of the back-end system for the management of payments and accounting, which will be based on ERPs. The cooperative gateway will be based on a proprietary architecture, already used by the Italian Banking System.

\subsection{Comparative Analysis}

The comparison among the different projects is carried out through a few analysis dimensions, aiming at describing the type and complexity of the project, and the adopted technologies, architectures and approaches.

The first set of dimensions concerns the complexity of the project; it consists of:

- Project schedule. This dimension provides the start time of the project, the length and the phase in which it currently is. The start time is often tied to the technology on hype in that period (as the projects are experimental, their aim is to try different new technologies).

- Involved administrations. As the projects aim at defining and deploying cooperative interfaces among previously non connected administrations, this dimension is used as a measure of the present and future complexity of the project. At the same time, the more the involved administrations are, the more the adopted architecture and engineering approach can be considered valid and applicable. In Table we show both the organizations currently involved and the expected final set.

As regards the type of project, from the previous dimensions it is possible to classify them in terms of proof-of-concept and core-business, meaning the first projects are more targeted to the experimentation of solutions in a small set of administrations, the latter ones concern more widespread applications and critical processes of the Italian PA.

Table shows this classification and the values of the described dimensions for each project. 
Table 1. Comparative analysis: complexity dimensions

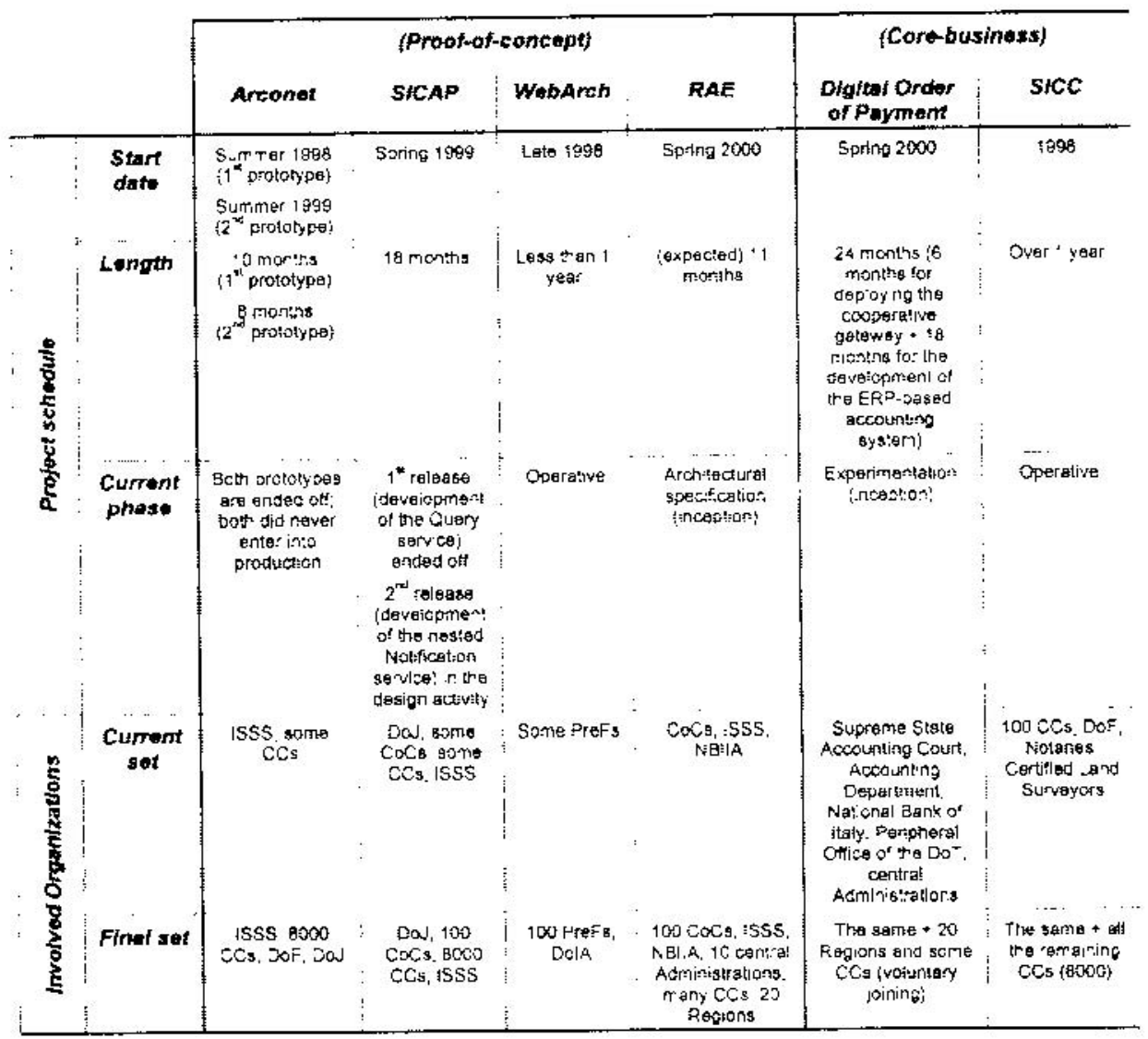

The second set of dimensions concerns the architectures and the cooperative models adopted by different projects:

- Cooperation Type. This dimension takes into consideration the different approaches that can be adopted for the design of Cooperative Gateways.

- Horizontal cooperation. In this case broad conceptual information and services are exported: they are not tied to specific business services and supporting applications, but rather they represent a general information model of the Domain. This approach aims at developing an "information bus" $\mathrm{n}$ top of which specific applications (such as portals) can be layered.

- Vertical cooperation consists in exporting coarse grained services aiming at supporting specific applications for specific processes (e.g. the Digital Order of Payment project).

- Interaction Type. The cooperation in the Italian PA can be targeted either to the Administration-to-Administration (A2A) exchange of data and services, or to the offering of services Administration-to-Customer (A2C). Customers can be 
classified into end-users (e.g. citizens, companies) and broker officials (such as tax accountants, notaries, etc.), who in many administrative processes act as intermediaries for end-users; therefore it is possible to identify Administrationto-EndUser (A2U) and Administration-to-BrokerOfficial (A2BO) sub-types of the $\mathrm{A} 2 \mathrm{C}$ interaction. The horizontal cooperation can support both $\mathrm{A} 2 \mathrm{~A}$ and $\mathrm{A} 2 \mathrm{C}$ types, as on top of the general information bus it is simple to develop specific client applications. Conversely in those projects adopting a vertical cooperation type, the choice about whether to support $\mathrm{A} 2 \mathrm{~A}$ or $\mathrm{A} 2 \mathrm{C}$ applications must be taken since the inception phase, as the specific nature of the produced interfaces.

- Reference Model. Prior to and during the projects, AIPA has defined a set of logical components as a reference model for the Cooperative Gateway: (a) Query and transactional Update services of the information asset of a Domain, (b) Event Notification service, (c) Control \& Management services (e.g. security, accounting, auditing, availability and fault control, QoS, etc.), (d) Cooperative Interfaces, which export data and application services, and (e) standard and possibly off-the-shelf middleware technologies, shown in Table with the specific suites of protocols used for the exchange of data and the invocation of services. As for the nature of the projects, not each system realizes all the logical components.

In Table the projects are described according to this set of dimensions. Finally the last set of dimensions concerns technological and architectural issues:

- Modeling Approach. This dimension is interesting for those projects adopting an object oriented approach to data/services modeling, whereas object wrappers integrate the back-end legacy systems to offer new cooperative interfaces: two different modeling techniques can be used, namely operation-based and concept-based (Mecella and Pernici 2001).

- Dealing with Legacy Systems. A basic issue is the pervasive presence of legacy systems in the Italian PA. About 100 different legacy systems currently operate in the Central PA, and among them at least $50 \%$ will be impacted by current and future cooperative projects. Different approaches to deal with them have been adopted in the projects.

- Middleware maturity. The projects adopt various technologies for the middleware layer, with different maturity levels. This dimension can qualitatively measure the risk of the project and its experimental nature, and therefore is correlated with the set of dimensions and the classification into proof-of-concept and core-business projects. In Table we provide the evaluated rating according to a three value scale. 
Table 2. Comparative analysis: cooperation model

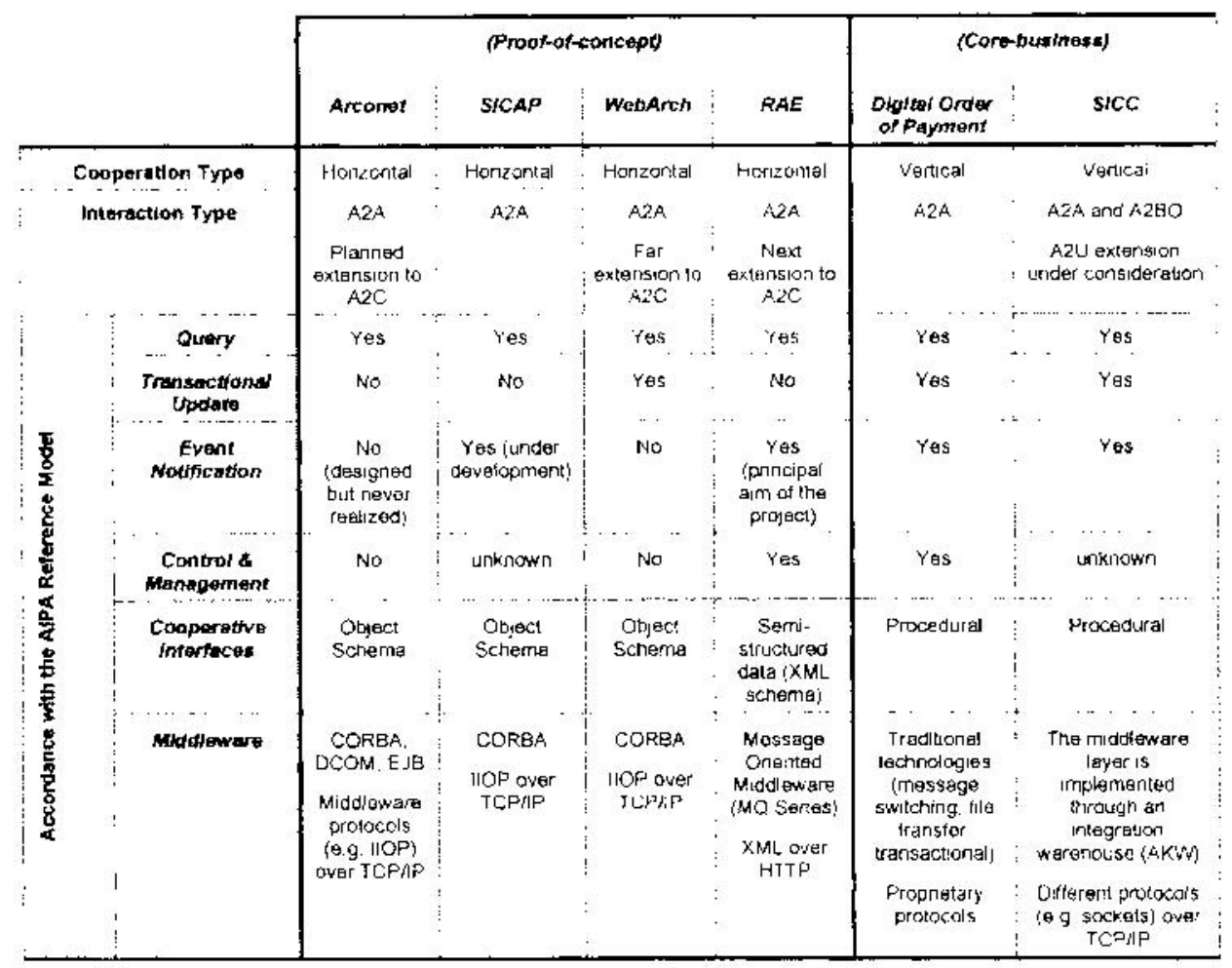

- Architectures. The adopted architectures can be roughly classified into three categories: (i) distributed object/component middleware-based, (ii) Web/XMLbased and (iii) traditional. This dimension is tightly correlated with the middleware dimension previously proposed.

In Table the projects are described according to this set of dimensions.

\subsection{Discussion}

It is interesting to draw some lessons from the previous comparison:

- The core-business projects (Digital Order of Payment, SICC), in order to reduce the risk due to their complexity, are adopting a more conservative approach, by using traditional technologies and engineering approaches such as vertical cooperation and procedural interfaces; conversely the proof-of-concept projects, with less critical constraints, are more targeted towards experimentation of on hype technologies and therefore their technological risk is higher (e.g. Arconet prototypes failed). Moreover the core-business projects are more targeted towards qualities such as security, performance and reuse of existing solutions, whereas the proof-of-concept projects are more targeted towards qualities such 
as interoperability, modifiability and reuse of the new cooperative interfaces (horizontal cooperation).

Table 3. Comparative analysis: architectural and technological dimensions

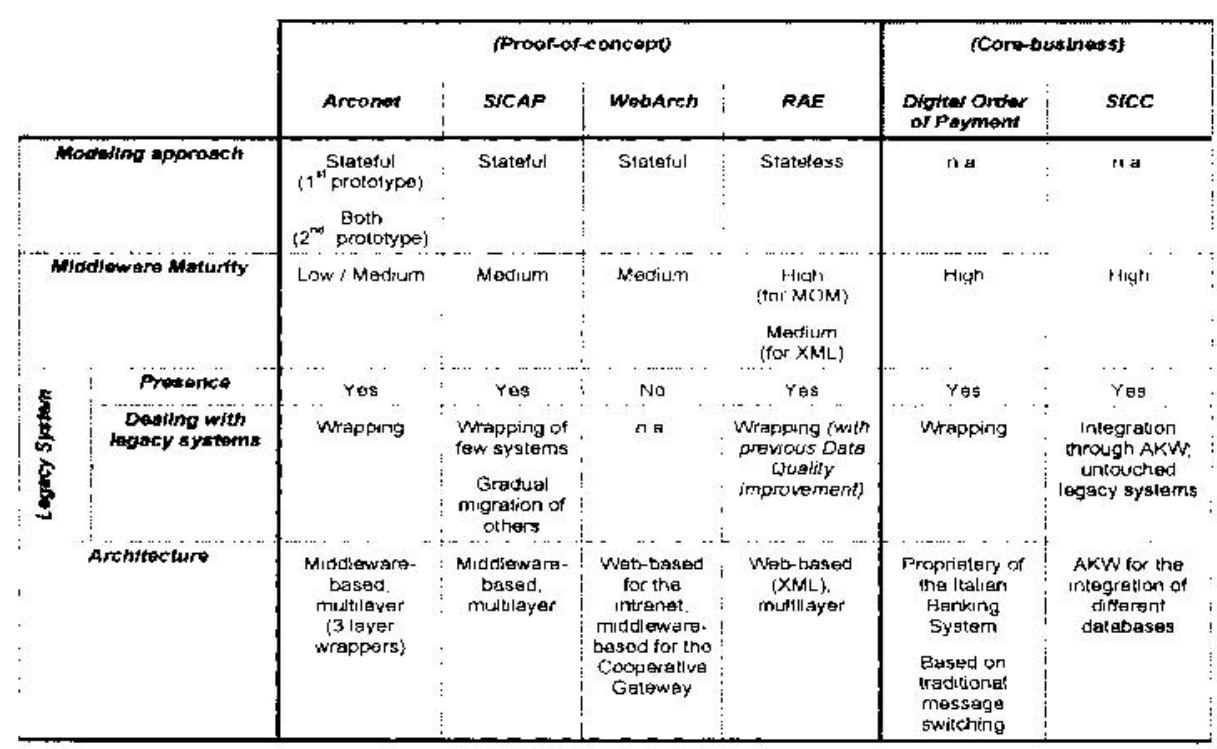

As regards the contexts in which to use simple data exchange vs. services, Webbased approaches vs. middleware-based ones, every time that the cooperation consists only in the simple exchange of data, a light approach (based on XML and standard Web technologies) seems more suitable, while, if the cooperation really consists in services (applications supporting processes) a middleware-based approach seems better. Our opinion is that the use of different engineering approaches (modeling, technologies, etc.) must be considered as evolutionary, not exclusive. Currently most of the projects require simple data exchange, and the risk involved by the middleware-based approach is sometimes too high; but the more the cooperation paradigm will spread and the BPR of the macro-processes will be pervasive, the more the cooperation will evolve from simple data query and exchange services towards the invocations of complex applications services. In the meantime the current middleware and Web technologies will probably merge, in order to resolve some issues and to converge towards a standard suite of protocols ${ }^{88}$.

88 One of the issues of the middleware-based approach is the security problem it creates (IIOP is not well transported through firewalls); whereas the Web-based approach (use of XML and HTTP) resolves this issue but lacks important middleware services. The trend is 
- The Cooperative Architecture relies on the integration of data and services from different Domains. It is fundamental to consider the data quality of the information to be integrated, in order not to thwart the cooperative effort (Missier, Scannapieco and Batini 2001). This leads to the consideration of specific activities in which to address such issues. Currently only the RAE project has considered these issues, including a data cleaning activity that precedes the development of the cooperative interfaces.

- The gained experience allows the identification of a possible meta-architecture for the Unitary Information System, which is shown in Figure.

o The Cooperative Interfaces logical layer is distributed over different Cooperative Gateways (each administration deploys its own interfaces on its Cooperative Gateways); this layer is on top of the single administrations' systems.

o Back-end layer. This layer is composed by the systems of the different administrations, often legacy systems. If a wrapping approach is adopted, the wrapping sub-layer could be itself multilayered, depending on organizational constraints and the conceptual mismatch between the legacies and the cooperative interfaces (Mecella and Batini 2000).

o The Client layer of the Cooperative Interfaces consists of portals, to offer services to citizens (A2C interaction), and of specific applications of single administrations, which integrate data and services exported by different Cooperative Gateways (A2A interaction). Portals will be on top of interfaces and/or applications of different administrations; this solution is suitable when the aim of the cooperation is to offer a single end-user interface (typically Web) to different administrations involved in a macroprocess. On top of the Cooperative Interfaces it is possible to have a further layer specializing the Cooperative Interfaces according to the specific client needs. 


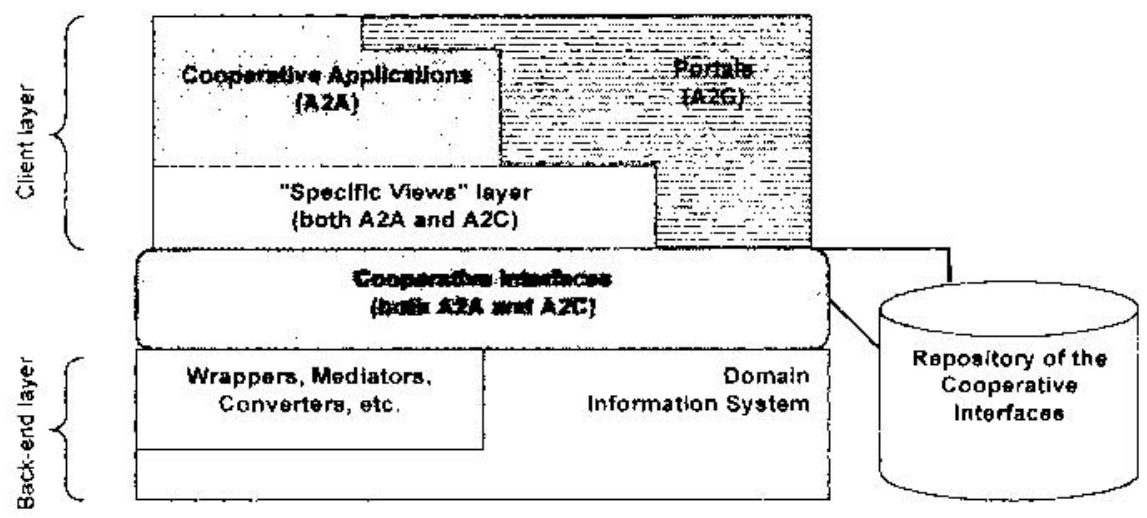

Figure 2. A meta-architecture for the Unitary Information System

- A repository of the Cooperative Interfaces exported by the different Domains, implied by the bottom-up development of such interfaces, need to be considered in the Cooperative Service. In this repository all the information about the interfaces (semantics, supporting conceptual schemas, handles for accessing them, etc.) must be provided.

- It is fundamental to set-up a joint development process, which is described in details in Mecella and Batini 2000 and Mecella and Batini 2001.

\section{CONCLUSIONS}

The constitution of AIPA, the initiative of the Unitary Network and, more recently, a new action plan directly set up by the Government (Italian Government 2000 ), are the main steps of the Italian approach to $e$-Government. The definition of a common Cooperative Architecture, based on Domains and Cooperative Gateways, will promote the cooperation at the application level, beyond the simple interoperability services already provided.

In the last few years many projects, both specific of a single administration and cooperative, have been carried out; in this paper the most relevant among the cooperative ones have been selected and compared, basing on a few dimensions, deriving some considerations and lesson learned.

The development of the Cooperative Architecture, just started in the Italian PA, is the coordination project in which almost all the projects for the $e$-Government will find their framework. Next years will show its evolution and the benefits it will offer to the customers, through a complete reengineering of the macro-processes servicing them; moreover it will offer the testbed for further research about Cooperative Information Systems. 


\section{REFERENCES}

Arcieri F., Cappadozzi E., Naggar P., Nardelli E., Talamo M. (1999): Access Keys Warehouse: a New Approach to the Development of Cooperative Information Systems. Proceedings of the 4th IFCIS International Conference on Cooperative Information Systems (CoopIS'99), Edinburgh, Scotland, 1999.

Autorità per l'Informatica nella Pubblica Amministrazione (AIPA): http: //www.aipa.it/english [4/ (link checked January, 1st 2001).

Batini C., Castano S., De Antonellis V., Fugini M.G., Pernici B. (1996): Analysis of an Inventory of Information Systems in the Public Administration. Requirements Engineering, vol. 1, no. 1, 1996.

Brodie, M.L. (1998): The Cooperative Computing Initiative. A Contribution to the Middleware and Software Technologies. GTE Laboratories Technical Publication, 1998. Available on-line: http://info.gte.com/pubs/PITAC3.pdf (link checked January, 1st 2001).

Mecella M., Batini C. (2000): Cooperation of Heterogeneous Legacy Information Systems: a Methodological Framework. Proceedings of the 4th International Enterprise Distributed Object Computing Conference (EDOC 2000), Makuhari, Japan, 2000.

Mecella M., Batini C. (2001): Enabling Italian e-Government through a Cooperative Architecture. In Elmagarmid A.K., McIver Jr. W.J. (eds.): The Ongoing March Towards Digital Government. Special Issue on Digital Government. IEEE Computer, vol. 34, no. 2, February 2001.

Mecella, M., Pernici, B. (2001): Designing Wrapper Components for e-Services in Integrating Heterogeneous Systems. To appear in VLDB Journal, Special Issue on e-Services, 2001.

Missier P., Scannapieco M., Batini C. (2001): Cooperative Architectures. Introducing Data Quality. Technical Report 14-2001, Dipartimento di Informatica e Sistemistica, Università di Roma "La Sapienza", Roma, Italy, 2001.

Mylopoulos, J., Papazoglou, M. (eds.) (1997): Cooperative Information Systems. IEEE Expert Intelligent Systems \& Their Applications, vol. 12, no. 5, September/October 1997.

Thompson C. (1993): Living an enterprise model. Database Programming and Design, March 1993.

U.S. Department of Commerce, National Bureau of Standards (1988): Guide to Information Resource Dictionary System Applications: General Concepts and Strategic Systems Planning. NBS Special Publication 500-152, U.S. Government Printing Office, 1988. 